\title{
PERSPECTIVAS DE UNA ANTROPOLOGÍA DE LA TÉCNICA
}

Amán Rosales*

Si hay algo de cierto en la vieja y noble idea, atribuida a Aristóteles, pero posiblemente de origen más antiguo, de que el asombro es el inicio de la especulación filosófica, quizá también lo haya en afirmar que la costumbre lo sea más bien de su final, no por cierto en el sentido de que inmerso en ella se llegue espontáneamente a la verdad sino todo lo contrario: el sentirse demasiado a gusto con ciertas situaciones (o con la forma de considerarlas) puede sin duda matar la curiosidad, sofocar el entusiasmo y enterrar definitivamente la capacidad de reflexión crítica. ¿Se habrá llegado a ese punto respecto de la tecnología en el mundo contemporáneo?

El tema del que se ocupa el presente ensayo incluye la presentación y discusión de dos enfoques propuestos por dos distinguidos, pero quizá poco conocidos (al menos en el ámbito de lengua castellana), filósofos alemanes contemporáneos, acerca del origen antropológico de la técnica, así como de la implicación de la tecnología, y su lugar en la cultura y la sociedad. Se trata de dos puntos de vista que buscaron distanciarse del acostumbramiento al fenómeno de la técnica.

Este trabajo es un intento de situar una misma tradición de problemas, una antropología de la técnica, el pensamiento de Arnold Gehlen (1904-1976) y el de Hans Sachsse (1906-1992). Mientras que las ideas del primero han tenido una influencia nada despreciables, en diver-

* Escuela de Estudios Generales, Universidad de Costa Rica. 


\section{AMÁN ROSALES}

sas áreas de las ciencias sociales, las más recientes del segundo autor todavía no disfrutan, injustamente, incluso en su propio país, de un estatus similar. Luego de la presentación de ciertos elementos teóricos básicos en los apartados (2) y (3), se procura evaluar de una forma objetiva y crítica la relevancia filosófica general de dicha tradición respecto de un tema específico: el de la autonomía de la tecnología. (4)

\section{2}

El punto de partida del enfoque antropológico propuesto por Arnold Gehlen, lo constituye la constatación del ser humano como un ser carencial, desposeído de un conjunto especializado de órganos o recursos instintivos que lo capaciten ventajosamente para el enfrentamiento eficaz, exitoso con su medio y con el resto de los seres vivos. Así, definido como un 'ser con carencias' o ‘ser carencial' -'Mängelwesen’1-, es decir, considerado desde el punto de vista de su pobreza instintiva y orgánica para ciertas funciones de subsistencia, el ser humano debe aprender a utilizar y aprovechar al máximo sus recursos físicos e intelectuales. Sólo de esa forma podrá paliar las carencias o imperfecciones corporales que lo ponen en franca desventaja frente a otros seres. En este sentido se explica la concepción de Gehlen acerca de la técnica; ésta se concibe como el medio por excelencia de que se ha valido la especie humana con el objeto de mitigar e incluso superar sus debilidades biológicas, sea mediante la sustitución, la complementariedad o el fortalecimiento máximo de condiciones más o menos preexistentes.

${ }^{1}$ Un término que Gehlen, en su obra antropológica principal: Der Mensch. Seine Natur und seine Stellung in der Welt de 1940, retoma de J. G. Herder, que lo utilizó en 1770. Cfr. K. Lorenz, Einführung in die philosophische Anthropologie, 1992, 2a. Darmstadt, Wiss. Buchges, 1992, 2a., p. 59-73, para una comparación entre ambos autores. W. Glaser, Soziales und instrumentales Handeln, 1972, Stuttgart, Kohlhammer. M. Deege, Die Technikphilosophie Arnold Gehlens, 1995, Hamburg, Kovac. A. Honneth, y H. Joas, Soziales Handeln und menschliche Natur, 1980, Frankfurt/M \& New York, Campus-Verlag. 
En el enfoque de Gehlen se continúan y profundizan ciertos lineamientos típicos de lo que ya se calificó de tradición antropológica respecto de la técnica. Dicha tradición alcanzó un momento decisivo (de hecho su comienzo 'oficial') en la obra pionera de Ernst Kapp (1808-1896), Grundlinien einer Philosophie der Technik. Zur Entstehungsgeschichte der Cultur aus neuen Gesichtspunkten (1877). Dada su influencia sobre el punto de vista gehliano, vale la pena detenerse brevemente en su contenido. En dicha investigación, Kapp resume sus ideas en torno al papel desempeñado por el progreso técnico en la cultura con su idea de la 'proyección de los órganos’ ('Organprojektion’), la auténtica base orgánica de la técnica. Sin embargo para Kapp los instrumentos y herramientas del mundo técnico no 'reproducen’ sin más el mundo orgánico específicamente humano. Con la técnica se efectúa una apropiación creativa de ciertos patrones orgánicos ingénitos en la constitución físico-mental del ser humano, que le permiten a éste volver con un poder incrementado sobre la naturaleza y sus procesos.

En cierto modo puede decirse que, desde el enfoque de Ernst Kapp, la técnica se haya potencialmente 'encarnada' en el organismo humano, a la espera de ser puesta en práctica y desarrollada como reacción a los estímulos ambientales. Una idea fundamental ya presente en dicho autor (retomada por Gehlen y Sachsse) es que el ser humano proyecta, consciente o inconscientemente, deseos y planes sobre una naturaleza concebida no sólo como depósito de posibilidades para el uso, sino también como una especie de ‘catalizador' que devuelve a su creador original, de una u otra forma, las obras técnicamente producidas. ${ }^{2}$

Esa doble dirección del proceso creativo, que involucra una unidad dinámica entre el ser humano y la obra técnica, es una intuición que Gehlen va a integrar, con las modificaciones del caso, en su propio esquema antropológico. Así explica Kapp el hecho de la fundamentación orgánica de ciertos instrumentos: "La riqueza de las creaciones espirituales brota, pues, de la mano, el brazo y los dientes. Un dedo doblado se convierte en un gancho, el hueco de la mano en un plato;

${ }^{2}$ Según la expresión de Deege, op. cit., p. 3. 


\section{AMÁN ROSALES}

en la espada, la lanza, el remo, la pala, el rastrillo, el arado y la laya, se observan diversas posiciones del brazo, la mano y los dedos, cuya adaptación a la caza, a la pesca, a la jardinería y a los aperos de labranza es fácilmente visible."3

De acuerdo a la explicación de Kapp (inspirada por Aristóteles), la mano representa el modelo original de todas las herramientas y el ejemplo más claro a favor de su teoría de la proyección orgánica: “La mano es por ende la herramienta natural de cuya actividad surge la artificial, la herramienta de mano. Ella ofrece en todos sus modos posibles de posición y movimiento, las formas orgánicas primigenias a partir de las cuales el ser humano ha imitado, inconscientemente, sus primeros necesarios utensilios.” " En forma muy similar a Kapp, Gehlen también recalcará la importancia de las manos, especialmente en su papel de antecesoras de las armas como otro ejemplo de proyección orgánica. ${ }^{5}$

Todo lo anterior pone en evidencia la visión marcadamente antropocéntrica de Kapp. El ser humano es el punto privilegiado de partida y punto culminante de llegada en todo el desarrollo de la vida. Éste es un aspecto muy importante de su teoría, pues incluye la idea del ser humano como el factor causal integral de la técnica, y de la cultura (en cierta forma también un 'aparato') como el reflejo de la propia naturaleza humana. Más aún, Ernst Kapp va tan lejos como para afirmar

${ }^{3}$ Citado por C. Mitcham, ¿Qué es la filosofía de la tecnología?, 1989, Barcelona, Anthropos, trad. C. Cuello Nieto y R. Méndez Stingl, p. 30.

${ }^{4}$ Citado por Deege, op. cit., p. 9-10.

${ }^{5}$ De ahí que tenga razón Alois Huning al destacar la analogía que puede establecerse entre el punto de vista de Kapp y el célebre principio de Protágoras del homo mensuram omnium. Según Huning, mientras que la frase original de Protágoras ha sido tradicionalmente interpretada subrayando su carácter subjetivista, la misma idea asociada ahora con las habilidades proyectadas del ser humano, según Kapp, adopta más bien un carácter hegelianamente objetivo, sobre todo en la medida en que toda novedad técnica ya está presente implícitamente en algún sector del organismo humano. Véase su "Deutungen vom 19. Jahrhundert bis zur Gegenwart”, 1990, en F. Rapp, Hg. Technik und Philosophie, Düsseldorf, vDI, 41-95. 
la identificación del progreso técnico, de factura humana, con la misma evolución cultural: "La historia entera de la humanidad, examinada con exactitud, se resuelve en la historia de la invención de herramientas cada vez mejores.” ${ }^{6}$ La perspectiva anterior parece asumir, quizá demasiado optimistamente, una relación de trasparencia y armonía entre el ser humano y sus obras, con las que se encuentra inextricablemente vinculado. Sin embargo, como se verá al final de este trabajo, dicha creencia resulta más bien ingenua al ser confrontada con la cruda realidad de un ambivalente desarrollo técnico o tecnológico.

Mediante la capacidad sustitutiva que la técnica pone a disposición del ser humano, el propio desarrollo técnico se convierte entonces, para volver al esquema de Gehlen, en condición necesaria de la evolución cultural. Con su posesión de distintas 'técnicas de descargo' ('Entlastungstechniken') para la supervivencia, el ser humano logra satisfacer las necesidades materiales básicas, tanto como otras menos urgentes, y así consigue enriquecer, simultáneamente, el propio acervo creativo que se acumula y transmite de generación en generación. Merced al ejercicio de aquellas ‘técnicas de descargo', el ser humano toma distancia paulatina de una naturaleza que se le presenta como hostil y amenazadora y comienza a tomar cuerpo gracias a la especialización creciente de ciertas funciones biológicas. Así, explicando la teoría de Gehlen, Heinrich Popitz escribe:

Las funciones más bajas son sustituidas por las más altas -las motoras lo son por medio de las sensoriales, las sensoriales por las intelectuales-, [este proceso se da] en tanto que las capacidades más altas se hacen cargo de las más bajas, es decir, por ejemplo, en tanto que podemos experimentar viendo lo que antes debíamos tocar, o en tanto que podemos recoger en la

\footnotetext{
${ }^{6}$ Una idea original de E. Reitlinger, según lo anota F. Rapp en su Filosofía analítica de la técnica, 1981, Buenos Aires, Alfa, trad. E. Garzón Valdés, p. 151.
} 


\section{AMÁN ROSALES}

imaginación experiencias del movimiento -hasta la descarga de la acción real gracias al lenguaje. ${ }^{7}$

Esta capacidad sustitutiva de funciones que el ejercicio de la técnica pone a disposición del ser humano, le posibilita alejarse cada vez más de su medio ambiente y moverse con mayor libertad en una segunda naturaleza, objetivamente artificial, confeccionada a su deseo y medida. La noción ya citada de ‘proyección de los órganos’ la extiende Gehlen al máximo con el propósito de que pueda cubrir las tres funciones básicas de la 'sustitución de órganos’ ('Organersatz’), la ‘descarga de órganos’ ('Organentlastung’) y la 'superación de los órganos’ ('Organüberbietung’). Los diversos artefactos técnicos se convierten, como lo describe Popitz, en auténticas ‘prótesis orgánicas’ que sirven al propósito tanto de mitigar las carencias y defectos corporales, como de incrementar artificialmente la eficacia de ciertas funciones específicas. Considérense ahora algunos ejemplos de Gehlen:

La piedra en la mano para golpear supera exitosamente al puño; el carro [y] el animal de monta nos descargan del movimiento de a pie y superan con mucho su capacidad. En el animal de carga el principio de la descarga se vuelve evidentemente claro. El avión, de nuevo, nos sustituye las alas que no nos han crecido y supera con mucho toda capacidad orgánica de vuelo. Algunos de estos ejemplos indican que hay una técnica muy antigua de lo orgánico: la domesticación, sobre todo la cría de animales es una técnica genuina que alcanza el éxito después de muchos experimentos. ${ }^{8}$

En realidad, el principio gehliano de la 'descarga' o del 'alivio de carga’ de las funciones orgánicas por mediación de la técnica determina

${ }^{7}$ H. Popitz, Epochen der Technikgeschichte, 1989, Tübingen, Mohr, p. 46.

${ }^{8}$ A. Gehlen, Anthropologische und sozialpsychologische Untersuchungen, 1986, Reinbeck b. H., Rowohlt, p. 94. (Esta edición contiene: Anthropologische Forschung, 1961 y Die Seele im technischen Zeitalter, 1957.) 
el curso general de la cultura. El proceso en su totalidad está guiado por una progresiva 'objetivación del trabajo humano' que, según Gehlen, se puede desglosar en tres etapas: en la primera, en la correspondiente al uso de herramientas, el ser humano todavía tiene que invertir un considerable gasto físico y mental propio; en la segunda, la de máquinas de fuerza y trabajo, lo que se objetiva y descarga es la fuerza física humana; finalmente, en la tercera y última etapa, la de los autómatas, se sustituye el esfuerzo mental y con ello se cierra el círculo de la sustitución del factor humano por parte de la técnica. ${ }^{9}$

Ahora bien, ¿cuál es el punto de partida de la antropología de Arnold Gehlen que tiene consecuencias más inmediatas para el tema de la técnica y que además se presenta como una premisa fundamental de sus reflexiones? Se trata de la consideración del humano como un ser primariamente actuante, es decir, Gehlen coloca la 'acción' ('Handlung') como el referente conceptual básico para los estudios antropológicos. ${ }^{10}$ La acción, dirigida en forma primordial a la transformación de la naturaleza con base en fines exclusivamente humanos, encierra en sí dos rasgos cuya formulación original Gehlen atribuye a Max Scheler:

Por un lado, la libertad original y radical que la acción humana posee comparada con los actos instintivamente determinados del resto de los animales. Dichos actos supeditan al resto de los animales a ciertos ambientes específicos de vital importancia para su supervivencia, a la vez que los incapacita para establecer contacto con otros ambientes Por otro lado, en la acción humana está implícita también la 'apertura hacia el mundo' ('Weltoffenheit'), la capacidad de escoger libremente, mediante una toma de distancia entre el yo y la realidad externa, el tipo de ‘mundo’ por conocer y modificar. Para Gehlen es importante recalcar que en el concepto de ‘acción’ está implícita una visión integral del ser humano.

No solamente ciertas ideas e intuiciones de autores como Herder, Kapp y Scheler resurgen en el esquema antropológico de Gehlen. Otros

${ }^{9}$ Ibid., p. 159-60. La clasificación la toma Gehlen de H. Schmidt.

${ }^{10}$ Cfr. para lo que sigue en este párrafo, 1986, p. 16-9. 
AMÁN ROSALES

pensadores pueden ser mencionados dentro de lo que es toda una tradición filosófica que ha ahondado en el carácter ‘abierto’ de la naturaleza humana. Se trata de un carácter que no se concibe como necesariamente negativo, sino como una veta potencial de logros creativos, consecuencia a su vez de un rasgo privilegiado de la especie: la libertad implícita en la acción. Desde ese punto de vista y según lo resalta especialmente por medio de varios ejemplos, H. Meyer, ${ }^{11}$ la idea de la capacidad de perfección que tiene el ser humano en razón, justamente, de su misma debilidad orgánica fue defendida con claridad por Diderot, para quien la perfectibilidad humana se relaciona directamente con su imperfección animal.

Sin duda mucho más significativa es la figura, dentro del idealismo alemán, de Fichte, quien ya había declarado que la característica básica del ser humano, a diferencia de la 'fijeza' instintiva de los animales, más bien reside en el estar abierto a un 'poder ser' guiado por los imperativos del espíritu. Según Fichte, el ser humano originalmente no es, sino que debe hacerse por sí mismo. Por ello es que también se ha escrito correctamente, a partir de lo anterior, que la idea fichteana de la esencia del ser humano como 'práctica intencional' está igualmente a la base del punto de partida gehliano y su propia concepción del ser humano como "un agente por naturaleza. No está por tanto ni metafísica ni biológicamente determinado: determina su propia esencia en tanto en cuanto se hace por sí sólo lo que es”. ${ }^{12}$

Pero es que el ser humano no solamente se hace a sí mismo, sino que desde el enfoque de Fichte también 'produce' el mundo, obra suprema de un Yo absoluto. ${ }^{13}$ Con relación a Gehlen hay otro punto de concordancia más claro con la perspectiva fichteana, pues ésta

${ }^{11}$ Cfr. para lo que sigue, H. Meyer, “Zur 'empirischen Philosophie’ Arnold Gehlens”, Philosophia Naturalis, 23, 1986, p. 407-8.

${ }^{12}$ R. Bubner, La filosofía alemana contemporánea, 1984, Madrid, Cátedra, trad. F. Rodríguez Martín, p. 256-7.

${ }^{13}$ Cfr. adicionalmente sobre este tema, H. Sachsse, Anthropologie der Technik. Ein Beitrag zur Stellung des Menschen in der Welt, 1978, Braunschweig, Vieweg, p. 194. 
también se manifiesta contraria “a una interpretación biológica del espíritu, es decir, contra una posición que concibe la razón únicamente como un fenómeno pragmático de compensación para una dotación orgánica o instintiva insuficiente de parte del ser humano. Desde [el] punto de vista [de Fichte] el espíritu constituye la esencia transanimal del ser humano". ${ }^{14}$

Según lo subraya Meyer, tales influencias filosóficas convergen en lo que podría llamarse un esbozo general del ser humano que Gehlen ha elaborado a partir de diversas fuentes, y que cuenta entre otros con los siguientes elementos constitutivos: la limitada capacidad y rendimiento de ciertos órganos, las potencialidades de desarrollo del espíritu, la variedad con que se expresa la acción humana y la ligazón armónica entre el ámbito corporal y el espiritual o intelectual. Desde la perspectiva de Gehlen, dichos elementos son los pilares fundacionales sobre los cuales la especie humana ha logrado establecer su sitio en el cosmos (privilegiado y precario a la vez), que de ningún modo hay que darlo por supuesto sino que ha implicado un laborioso esfuerzo de creación cultural e institucional.

El resultado final de esa actividad interventora y transformadora del ser humano sobre la naturaleza no es para Gehlen sino la cultura: el producto de la acción humana sobre la naturaleza que representa, a su vez, una suerte de segunda naturaleza tan irrenunciable como la primera. Con la cultura, el ser humano se ha creado una naturaleza sustituta de la primera, a su propia medida, orgánica y vinculada a sus propios intereses vitales. Gehlen se refiere, a una 'esfera de la cultura' ('Kultursphäre') en tanto que concepto abarcador de todo el conjunto de condiciones creadas por el ser humano, es decir, establecidas a partir de la transformación de la naturaleza.

Es llamativo constatar cómo no habría en principio, desde dicha perspectiva límites ‘naturales’ al proceso expansivo del dominio humano sobre la naturaleza. Gehlen más bien insiste en los límites 'técnicos' de la acción humana. Es decir, los obstáculos por vencer dependen de

\footnotetext{
${ }^{14}$ Meyer, op. cit., p. 407.
} 


\section{AMÁN ROSALES}

la misma capacidad humana para mejorar y hacer avanzar su capacidad técnica sobre la realidad, por eso, "los límites de la expansión humana son puestos, ante todo, por los medios intelectuales [Denkmittel] y objetivos o materiales [Sachmittel] [disponibles para dicha empresa]”. ${ }^{15}$

Puede decirse que la visión final de Gehlen con relación al desarrollo técnico y su vinculación con la cultura en general es básicamente optimista. ${ }^{16}$ En este sentido es importante destacar su severa reacción contra Rousseau y su agria crítica de la cultura. Esto es, desde el punto de vista de Gehlen hay que reconocer y aceptar la urgencia de un ‘jretorno a la cultura!' como parte de un programa generalizado que podría calificarse, a partir de sus mismas ideas (aunque la expresión no es suya), de auténtica 'superación de la naturaleza'. Resulta inequívoca la animadversión con que Gehlen se refiere a un presunto 'estado de naturaleza' de la humanidad; para él, la noción de 'estado natural' apunta a una situación de 'caos', ni más ni menos que ante la "Cabeza de Medusa frente a cuya mirada uno se paraliza. La cultura [por el contrario] es lo improbable, a saber el derecho, lo civilizado, la disciplina, la hegemonía de la moralidad.”"17

Tres son, especialmente, los rasgos que sobresalen en la perspectiva antropológica de Sachsse: en primer lugar, la acentuación del enfoque empírico y naturalista (compartido por Gehlen y Kapp) del marco teóri-

${ }^{15}$ Anthropologische, op. cit., p. 48. No dejan de ser sorprendentes las afirmaciones de Gehlen, pues, como ha indicado H. Stork, ellas parecen asumir, de modo más bien ingenuo, que la ilimitada voluntad humana de dominio tiene su contrapartida en una naturaleza concebida como un depósito igualmente inagotable o ilimitado de recursos y fuerzas, (Einführung in die Philosophie der Technik, 1977, Darmstadt, Wiss. Buchges, p. 149).

${ }^{16}$ Sin embargo, algunas precisiones sobre este juicio se reservan para el apartado final de este trabajo.

${ }^{17}$ Ibid., p. 60. 
co general para el estudio del ser humano en su relación con la técnica. En segundo lugar, en la obra de Sachsse también es llamativa la insistencia en la responsabilidad ética, en tanto que acompañante de la propia fundamentación antropológica de la técnica. De hecho, Sachsse califica de 'novedad absoluta' en la historia el hecho de que en la actualidad el ser humano haya asumido el papel de corresponsable de su existencia en el marco general de la naturaleza. Conciencia de que de las medidas que hoy tomemos dependerá crucialmente cómo viviremos mañana y cómo vivirá nuestra descendencia. ${ }^{18}$ En tercer lugar, un punto por igual significativo es la controversia, tácita o expresa por parte de Sachsse, contra intentos de identificar, sin más, el desarrollo técnico con la evolución biológica general.

Ahora bien, como punto de partida inmediato para la exposición, la que de momento se concentrará en el primer rasgo mencionado, considérese el siguiente pasaje en el que Sachsse formula algunas de sus tesis fundamentales, que al emparentarlo con otros autores lo inscriben en una reconocida tradición antropológica: “La técnica, que para nosotros está tan cerca y sin embargo tan lejos, de la que hablamos como si fuese una cosa en sí, no es un poder demoníaco, extraño, que nos avasallará o liberará, sino que es una parte de nuestra esencia, un miembro de nuestra naturaleza, figurativamente hablando un órgano de nuestro cuerpo, pero que nosotros aún consideramos como un trozo extraño, porque todavía no lo hemos reconocido como propio.”19

Lo anterior da pie para resaltar cómo para Hans Sachsse (al igual que para Kapp y Gehlen), el enfoque del fenómeno de la técnica como elemento constitutivo de la naturaleza humana lo habilita como medio legítimo para la autocomprensión humana. Su propuesta va en el sentido de reconocer la estrecha vinculación de la filosofía de la técnica con la antropología (empírica y filosófica), que Sachsse captura conceptualmente en su idea de una antropología de la técnica (título de

${ }^{18}$ Cfr. H. Sachsse, “Zur Anthropologie der Technik”, en A. Menne, Hg. Philosophische Probleme von Arbeit und Technik, 1987, Darmstadt, Wiss. Buchges, 122-35.

${ }^{19}$ Anthropologie, op. cit., p. 6. 


\section{AMÁN ROSALES}

su obra principal). Se trata de un enfoque que intenta mostrar que así como la técnica es parte esencialmente constitutiva de la naturaleza humana, así también “una filosofía de la técnica, desde una perspectiva antropológica, comprende su tarea de una manera muy estrecha y la yerra, cuando no se entiende también como una filosofía del autoconocimiento". 20

Sin embargo, la tarea de entender la técnica, que por lo visto también entraña la tarea de autoconocerse como ser humano, no resulta fácil de llevar a cabo. La razón de ello la encuentra Sachsse en el hecho de que con 'la técnica’ no se señala a un ámbito independiente, desligado de otras áreas como la ciencia, el arte, la política, la religión y la economía. Más bien, Sachsse asegura que la técnica concurre en todas esas áreas como 'una forma del comportarse' específico de cada una. La idea es que, en principio y en lo primordial, la técnica se encuentra como instrumento o medio neutral de ayuda, independientemente de las metas anticipadas, siempre actuante en todo cuanto el ser humano realiza. Pero además, lo notable y paradójico en todo ello es que aun cuando la técnica se conciba solamente como un instrumento colaborador, como un simple medio neutral para las intenciones humanas, los actos técnicos o tecnológicos influyen de una manera tan decisiva, profunda y particular en el modo de vida y en la conducta humana general, que su manejo y control se torna con frecuencia engorroso, problemático e incluso peligroso.

Así, pareciera que a partir de lo comentado en el párrafo anterior, el obstáculo mayor para un examen objetivo del fenómeno técnico curiosamente consiste en su misma presencia constante en la cultura humana desde sus mismos inicios. Dicha presencia parece que tiende a disimular el enorme efecto de la técnica sobre la cultura, pues ciertamente se podría argumentar, que lo que está en todas partes parece no estar en ningún sitio en particular; por lo que no parece urgente dedicar tiempo ni atención a un asunto tan banal y cotidiano como el de la relación humana con la técnica. Tal actitud de indiferencia o desinterés resulta

${ }^{20}$ Ibid., p. 6. 
inaceptable para Sachsse. Desde su punto de vista la filosofía de la técnica se enfrenta a una empresa apremiante, pero también difícil y compleja, pues debe preguntarse, según su formulación, “cómo la técnica ha surgido desde la humanidad y junto a ella, cuáles estructuras particulares le son propias en sus distintos estadios de desarrollo, cómo estas estructuras afectan la forma de vida del ser humano y cómo está el ser humano en condiciones de ordenar nuevamente dicha influencia en su vida como un todo". ${ }^{21}$

Hans Sachsse caracteriza la técnica como una forma particular de acción, una en la que se elige un rodeo ('Umweg') “porque el objetivo es más fácil de alcanzar por medio de ese rodeo”. ${ }^{22}$ La acción técnica se presenta, en efecto, como un medio entre la intención y la meta deseada, que de otro modo resultaría más difícil de conseguir. Desde luego, según casos específicos, los rodeos pueden ser más sencillos o complicados, etc. Sin embargo, Sachsse arguye que la idea misma de que el rodeo puede ser la vía más expedita para lo que se desea obtener está apoyada en la propia estructura orgánica general de la naturaleza. De acuerdo con su enfoque, se puede encontrar en el nivel básico y elemental de las reacciones químicas un ejemplo especialmente adecuado para concebir la técnica como un rodeo. Se trata del papel desempeñado en tales procesos por el catalizador, que se caracteriza por ser, "una sustancia que acelera la velocidad de la reacción, por así decirlo, gracias a su pura presencia, sin que ella misma cambie”. ${ }^{23}$

${ }^{21}$ Ibid., p. 7.

${ }^{22}$ Ibid., p. 9. Para versiones complementarias, aunque más breves de esta idea, cfr. sus obras citadas de 1972 y 1987. Alois Huning, en su reseña de Sachsse (1978) escribe correctamente: "Merece ser retenido el hecho de que Sachsse es el autor de esta tesis del rodeo, la que tanto contribuye a la comprensión de la técnica y sus efectos, sobre todo porque hace inteligible las posibilidades de desarrollo -y con ello muchas consecuencias involuntarias de la técnica- entre las situaciones iniciales y las metas” (Ch. Hubig, A. Huning, und G. Ropohl, Hrsg. Nachdenken über Technik: die Klassiker der Technikphilosophie, 2000, Berlin, Sigma, p. 323).

${ }^{23}$ Ibid., p. 10. 


\section{AMÁN ROSALES}

Los catalizadores resultan por lo general más eficaces desde el punto de vista químico, en la medida que pueden ser aplicados a procesos divididos en etapas cada vez más diferenciadas y específicas: “Con el surgir de la vida se configuran estructuras causales altamente complejas que controlan el metabolismo, el crecimiento y la multiplicación, y que superan con creces, en su grado de diferenciación, nuestros aceleradores artificiales de reacciones, al mismo tiempo que provocan una considerable aceleración de los procesos biológicos.”24

Un patrón muy semejante en el aprovechamiento de la estrategia catalizadora puede encontrarse según Sachsse en niveles más altos de la evolución orgánica, donde distintas etapas organizadas de modo diferenciado procuran a sus poseedores, establecidos en medio ambientes específicos, ciertas ventajas y beneficios para la consecución más rápida de objetivos en su lucha por la reproducción y la supervivencia. Precisamente, arguye Sachsse, la superioridad manifiesta de ciertas estructuras en la competencia evolutiva se basa en la puesta en práctica de una eficiente división del trabajo. ${ }^{25}$ En ese sentido, aunque la reproducción directa de organismos inferiores por partenogénesis parece ser el camino más fácil y rápido, lo cierto es que la reproducción sexual, con sus largos rodeos biológicos y culturales al final resulta un proceso cualitativamente superior al primero por las posibilidades de combinación de rasgos hereditarios, “de modo que a partir de un idéntico depósito genético, se producen descendientes más fuertes”. ${ }^{26}$

Sachsse argumenta que, mientras que la naturaleza debe probar y reintentar en formas muchas veces penosas y lentas los mejores medios hasta alcanzar las vías más rápidas de reacción, los mejores rodeos, el ser humano puede, en virtud de su equipamiento racional y gran poder imaginativo, captar y almacenar intelectualmente las conexiones percibidas en la naturaleza y aprovecharlas al máximo. De ese modo, el

${ }^{24}$ H. Sachsse, Technik und Verantwortung. Probleme der Ethik im technischen Zeitalter, 1972, Freiburg, Rombach, p. 52.

${ }^{25}$ Cfr. Anthropologie, op. cit., p. 11-4.

${ }^{26}$ Technik, op. cit., p. 53. 
lento procedimiento de la prueba y el error puede acelerarse gracias al recurso intelectual que permite anticipar situaciones problemáticas y sus posibles soluciones. Aquí se muestra en forma patente una tesis central del enfoque antropológico de Sachsse: que "el desarrollo de la técnica humana está ligado de modo inmediato con el despertar [de la] consciencia, con el desarrollo [de la] capacidad imaginativa y con la aptitud para tener experiencias, acumularlas y utilizarlas ingeniosamente de nuevo". ${ }^{27}$

En un trabajo anterior, esta misma idea con la que Sachsse de nuevo se vincula a intuiciones similares de Kapp y Gehlen se resumía de manera semejante: "Hemos calificado como técnica la elección pensada [de un] rodeo, y por eso podemos decir ahora que el instante del nacimiento de la consciencia es al mismo tiempo el de la técnica." ${ }^{28}$ El rasgo característico del progreso técnico se pone de manifiesto, según Sachsse, en la 'largura de los rodeos', es decir, en la habilidad humana para conectar distintos pasos intermedios con los cuales alcanzar más exitosa y provechosamente las metas previstas. El autor propone dos ejemplos:

Por una parte, mientras que en la utilización de los primeros martillos se expresa (mediante el golpe directo mismo) toda la inmediatez del fin esperado, en su producción ulterior con el paso del tiempo, progresivamente más compleja y onerosa (por ejemplo en grandes industrias robotizadas dedicadas al tratamiento de maquinaria pesada), se observa un alejamiento cada vez mayor respecto de los propósitos iniciales del primitivo utensilio. La complejidad posterior obedecerá a las condiciones requeridas para una mayor eficiencia o calidad de las acciones y los resultados De otra parte, en el caso de la domesticación del fuego, los usos posteriores de la técnica han dejado atrás la inmediatez del primer efecto deseado dando lugar a la consolidación de prácticas culturales, como el desarrollo de la vida familiar, de más amplio alcance social.

${ }^{27}$ Anthropologie, op. cit., p. 15.

${ }^{28}$ Technik, op. cit., p. 53. 
AMÁN ROSALES

Conforme avanza la cultura los fines previstos deberán alcanzarse siguiendo un rodeo más largo y complejo, al menos proporcionalmente a la dificultad y magnitud misma de las metas, las que, obviamente, deberán estimarse como valiosas o dignas de ser perseguidas. Por ejemplo, según Sachsse, toda la empresa relacionada con el alunizaje estadounidense de 1969 absorbió, durante casi una década, una cantidad enorme de recursos humanos y materiales, así como también necesitó de la integración de diversas disciplinas y campos de la ciencia, la tecnología y la industria en general. ${ }^{29}$

Sachsse propone aún varios rasgos adicionales que complementan su caracterización original de la técnica como estrategia utilizadora de rodeos: ${ }^{30}$ (a) El actuar técnico representa desde sus inicios un actuar social, pues supone, al depender de la capacidad humana de comunicación, la cooperación y distribución de tareas en una colectividad. (b) Mediante el ejercicio reflexivo de su capacidad técnica, transformadora de la realidad, el ser humano descubre que la estructura de la naturaleza resulta sorprendentemente afín y compatible con dicha capacidad de acción técnica. Ello no es tan extraño tomando en cuenta que dicha estructura está determinada por la utilización de rodeos, incluso en los más bajos niveles moleculares. De allí que el propio acto técnico, materializado en el medio mismo, independientemente de un propósito utilitario, adquiera con frecuencia un rango privilegiado, en tanto que acto promotor de la realización humana espiritual. Sachsse insiste en señalar cómo la mera posibilidad del hacer otorga a la actividad técnica un peso propio, en virtud del cual la creatividad puede muchas veces manifestarse sin una preocupación inmediata por la utilidad de lo creado. Este entusiasta impulso creativo es un factor muy importante, que hay que en tomar en cuenta al considerar los distintos motivos que influyen en el avance continuo de la técnica. ${ }^{31}$

${ }^{29}$ Cfr. sobre los ejemplos citados, Anthropologie, op. cit., p. 15-6.

${ }^{30}$ Cfr. ibid., p. 16-7.

${ }^{31}$ Cfr. sobre los distintos factores que inciden en el desarrollo técnico, de Arnold Pacey, The Maze of Ingenuity. Ideas and Idealism in the Development of Technology, 1992, 2nd. ed., Cambridge, Mass., The MIT Press. 
UNA ANTROPOLOGÍA DE LA TÉCNICA

Otro rasgo importante relacionado con la creatividad técnica es (c) la capacidad de aprender, que representa un enorme salto cualitativo en el conjunto de la evolución, pues justamente otorga las premisas centrales del progreso científico-técnico. La dirección de la evolución cultural está determinada por la flexible capacidad de adaptación del cerebro humano a las exigencias del medio ambiente y del entorno cultural. Y para Sachsse lo que mejor se aprende y transmite es en esencia la técnica misma: "Se trata del descubrimiento y aprovechamiento de la posibilidad ofrecida por la naturaleza de alcanzar más fácilmente un fin por medio de la inserción de etapas intermedias.”32

De acuerdo con la interpretación de Sachsse, la habilidad de aprender, conservar, transmitir y aplicar metódicamente el conocimiento sitúa al ser humano en un lugar muy particular de la evolución. Su flexible adaptación al medio, su ‘apertura al mundo’ le separan radicalmente de los otros pobladores del planeta. De ahí precisamente que la capacidad de aprendizaje le otorgue al desarrollo técnico características distintivas por comparación con la evolución biológica.

Aunque Sachsse insiste en el modo occidental de realización de la capacidad técnica, especialmente en su vinculación con la ya destacada capacidad de aprendizaje del ser humano, también dedica interesantes reflexiones a otra posibilidad de su interpretar antropológica. Se trata de una alternativa a la técnica mayormente extrovertida y quizá típica de Occidente: la técnica introvertida de Oriente tal y como se puede observar, entre otras corrientes, en el budismo y el budismo-zen.

\section{4}

Reseñadas las características básicas de la tradición antropológica en dos de sus autores más representativos, procederemos a examinar con mayor detalle su postura con relación al tema indicado al comienzo de este ensayo: el de la autonomía de la tecnología o determinismo

${ }^{32}$ Ibid., p. 37. 
AMÁN ROSALES

tecnológico. Considérese, en primer lugar, el punto de vista de Arnold Gehlen.

¿Comparte Gehlen la idea de un desarrollo tecnológico autónomo, que parece avanzar casi al margen de voluntades y deseos humanos? La respuesta es afirmativa, dado que su perspectiva destaca cómo las actuales sociedades industriales se hayan regidas por una gigantesca superestructura, constituida por tres elementos: la propia técnica o tecnología, las ciencias naturales en su versión matemática-cuantitativa y la forma capitalista (hoy se diría, además, neoliberal y globalizada) de producción. Tal y como lo aclara H. Stork, el grupo de tres elementos supone para Gehlen una completa 'unidad funcional', ${ }^{33}$ de modo que con relación, por ejemplo, a la gran industria mundial de producción de medicamentos, no tiene sentido preguntar qué es lo primordial, si la investigación ‘pura’ propiamente dicha, la producción tecnológica o, en fin, la organización industrial de los productos y su comercialización final.

Dicha superestructura aparece adicionalmente como un gigantesco organismo planetario de movimientos no enteramente predecibles ni controlables. Su presencia representa un hecho insólito que distingue claramente a la cultura industrial contemporánea de sus predecesoras. ${ }^{34}$ En este punto Gehlen cita un texto donde Werner Heisenberg compara la dinámica de la técnica con un proceso biológico, “en el que las estructuras dispuestas en el organismo humano se transmiten en medida cada vez más amplia al medio ambiente del ser humano; un proceso biológico que justo en cuanto tal se ha sustraído del control humano". ${ }^{35}$ (Pronto se verá cómo Hans Sachsse combate vigorosamente la presunta simetría entre la evolución técnica y la biológica.)

\section{Stork, p. 139.}

${ }^{34}$ Cfr. A. Gehlen, “Anthropologische Ansicht der Technik”, en H. Freyer, J. Chr. Papalekas y G. Weippert, Hg., Technik im technischen Zeitalter. Stellungnahmen zur geschichtlichen Situation, 1965, Düsseldorf, Verlag Joachim Schilling, p. 104-5.

${ }^{35}$ Ibid., p. 113. 
Según Gehlen, con aparente autonomía los tres elementos de la superestructura han conformado una relación de interdependencia recíproca provocando que la humanidad "se coloque en la posición del capitán de un navío tan fuertemente construido de hierro y acero, que la aguja magnética de su compás únicamente apunta a la masa de hierro del barco" ${ }^{36}$ Esta imagen utilizada por Gehlen va al corazón del determinismo tecnológico: el ser humano ha creado algo que sigue ahora su propia dinámica de desarrollo, insensible a los lamentos de sus diseñadores, que podrán mejorar o eliminar alguna pieza mas no son capaces de ‘desconectar' del todo a la ‘Máquina’, ¡tan inabarcable se ha vuelto en su conjunto y tan vitalmente conectado a ella se encuentra el resto de la sociedad!

Hay todavía un punto adicional que hace más densa la atmósfera de pesimismo cultural, pues para Gehlen con el entronizamiento definitivo de esa superestructura funcionalmente autónoma, las sociedades industriales parecen haber alcanzado también un límite teórico o intelectual. Se trata de un punto límite, en el que todas sus opciones de perfectibilidad convergen en un estado de 'cristalización cultural', en una suerte de 'fin de la historia' o 'poshistoria', una fase en la que todas las posibilidades civilizadoras, todas las opciones ideológicas posibles se habrían agotado en una especie de Estado científico-tecnológico mundial. ${ }^{37}$ Desde esta perspectiva ideológicamente desencantada

${ }^{36}$ Citado por Glaser, p. 21.

37 Cfr. sobre el tema de la 'poshistoria', A. Gehlen, “Über kulturelle Kristallisation”, Studien zur Anthropologie und Soziologie, 1961, Neuwied und Berlin, Luchterhand, 1971, 2a., p. 283-300. Además, sobre el importante fenómeno de la 'cristalización cultural' y sobre la defensa a ultranza de las instituciones como garantes de estabilidad cultural, cfr. Stork, op. cit., p. 139, 142. Ideas como las anteriores, relacionadas con la defensa de las instituciones, han dado pie para calificar de pesimista y fatalista la perspectiva de Gehlen, incluso de un pensamiento contrailustrado y excesivamente conservador. En ese sentido, quizá haya que coincidir con Herbert Schnädelbach cuando afirma que estar "ilustrado acerca de la Ilustración significa, para Gehlen, tanto como saber que ella ha llegado a su fin. La fe en la bondad 


\section{AMÁN ROSALES}

habría que optar por conservar lo bueno obtenido antes que malograrlo buscando la quimera de la perfección política.

¿Implica lo anterior una aceptación implícita de la idea de que cuanto más cambian las cosas, más siguen siendo las mismas? Ése parece el sentido, por ejemplo, de la interpretación de Rüdiger Bubner, para quien a pesar de que "la historia está incesantemente produciendo cambios, [para Gehlen] la esencia de los seres humanos cambia poco con ella. Las esperanzas de progreso que anticiparon una humanidad nueva o completamente diferente en el futuro aparecen necesariamente como dudosas”. ${ }^{38}$ De ahí que Gehlen, después de todo coherente con su creencia en coacciones legales objetivas ('Sachzwänge') que determinan, económica y tecnológicamente el transcurrir de las sociedades capitalistas del presente, cifre gran parte de sus esperanzas en un último reducto de reacción, y clame porque el individuo recupere un sentido de ascetismo, de autocontención frente a la tentación del consumo y la masificación.

En el caso de Hans Sachsse, su aproximación al tema del determinismo tecnológico comienza con la siguiente interrogante: ¿de dónde surge la idea de una técnica desbocada y con poder casi total de autodeterminación? Él cree que la misma aparición de la consciencia, fundamental para el avance continuo de la acción técnica, tendió no sólo a separar sino a enfrentar al ser humano y sus recursos instrumentales con la naturaleza. El pensamiento griego, señala Sachsse, expresó tal dicotomía entre las obras humanas y las naturales con las nociones de Techne y Physis. Una consecuencia de esa situación se expresa en la actitud del hombre de la calle del presente, para quien la suma total de artefactos que le rodea asume el cariz de un mundo independiente, ‘artificialmente’ extraño.

natural del ser humano, en derechos humanos eternos e inajenables, en 'la' historia y ‘el’ progreso es para él la imagen moderna de la superstición, contra la que una vez combatió el movimiento ilustrado burgués de los siglos XVII y XVIII" (“Nachwort”, en Gehlen, Anthropologie, op. cit., p. 268-9).

${ }^{38}$ Bubner, op. cit., p. 258. 
UNA ANTROPOLOGÍA DE LA TÉCNICA

Es con relación a ese sentimiento de impotencia ante el proceso del desarrollo tecnológico actual, que tiene sentido la crítica de Sachsse al intento de unificarlo con la evolución biológica de los organismos, fortalecida por la utilización de vocablos tomados del ámbito de lo orgánico que son trasplantados a otras esferas de la cultura, como 'crecimiento', 'desarrollo’, 'fecundidad', ‘maduración', 'organización’, etc. Igualmente popular es la inclinación a concebir el desarrollo técnico en su conjunto "como el resultado de una suma de fuerzas impulsivas biológicas (...), como un proceso natural que se sustrae a la influencia humana". 39

Sachsse rechaza vehementemente la idea de un determinismo tecnológico total, porque si bien es cierto que la identificación de la evolución biológica con la técnica encierra cierta plausibilidad, también entraña una peligrosa ilusión. Cree que concebir la técnica como un organismo vivo, cuyo desarrollo se da en forma espontánea y al margen del ser humano, no es ni más ni menos que ‘un mito moderno' en el que se proyecta hacia fuera el propio comportamiento para concebirlo, posteriormente, como una fuerza actuante con impulso propio, extraña y amenazadora: “esta mitologización de la técnica, ligada con frecuencia su demonización, obstaculiza no sólo el acceso a su comprensión precisa, sino que también es peligrosa porque con ello el ser humano renuncia al control de un instrumento del que depende para su existencia". 40

Por contraste con posiciones fatalistas de la historia y de determinismo tecnológico, Sachsse resalta la capacidad y deber del ser humano de asumir consciente y responsablemente la dirección del desarrollo tecnológico. En especial ataca las que pretenden justificar (aduciendo o sugiriendo que el progreso tecnológico continúa con medios más productivos y eficaces la evolución biológica en su conjunto) el carácter inevitable de ciertos avances. Considera que el reconocimiento de las raíces antropológicas de la técnica no implica, ni mucho menos, la

${ }^{39}$ Anthropologie, op. cit., p. 34.

${ }^{40}$ Ibid., p. 35. 
AMÁN ROSALES

aceptación de una supuesta 'lógica interna’ en el progreso tecnológico concebido como la simple contrapartida cultural del desarrollo espontáneo o fortuito de sucesos naturales.

Para Sachsse la disposición hacia la técnica, la búsqueda de rodeos cada vez mejores para la obtención de ciertas metas, en fin, la proyección extrovertida del ser humano y su consciencia en los procesos naturales no entraña en absoluto la neutralidad intocable de los recursos y medios técnicos empleados en la interacción con la realidad. Valga agregar, muy brevemente, que este autor entrevió la posibilidad de reacción contra la tecnificación total, en la alternativa de remozar no sólo la interioridad (lo que lo acerca a la apelación gehliana al poder del individuo), sino también la solidaridad y el impulso humano de cooperación en el marco de una sociedad de corte socialista.

Podría establecerse una significativa coincidencia entre Gehlen y Sachsse, en la medida que para ambos es vital rescatar la interioridad humana, la 'personalidad' integral del ser humano según Gehlen, de su apropiación por parte de la gigantesca superestructura económica y tecnológica del presente. Tal exigencia armoniza muy bien con la del propio Ernst Kapp, en el sentido de que el ser humano debe recuperar el control sobre los peligrosos productos que su misma consciencia, volcada al exterior ha creado a veces sin quererlo. Como sugiere Deege, ${ }^{41}$ la situación actual requeriría, para Kapp seguramente en coincidencia con Gehlen y Sachsse, de la gestación adicional de una vigorosa consciencia de responsabilidad colectiva, institucional de cara al porvenir y las futuras generaciones.

En este punto se pone de manifiesto el resultado paradójico, pero quizá no del todo sorprendente, a que conduce un intento final por valorar la antropología de la técnica tanto en Kapp como en Gehlen y Sachsse. Las posibilidades de solución que ofrece dicha tradición para enfrentar, por sí sola, el problema del determinismo tecnológico son ambiguas.

${ }^{41}$ Deege, op. cit., p. 22. 
UNA ANTROPOLOGÍA DE LA TÉCNICA

Desde una lectura más optimista y favorable, cabría resaltar que tal vez ninguna otra perspectiva filosófica ha insistido tanto como la representada por estos autores, en la necesidad de considerar las raíces antropológicas naturales de la técnica, con lo que se coloca al ser humano en un sitial privilegiado de dominio y control sobre sus creaciones. Ésa es en principio ${ }^{42}$ la faceta más benévola de la tradición antropológica respecto de la técnica.

Pero desde una lectura más pesimista y recelosa, cabría más bien lamentar que el carácter intencional de la producción técnica ('proyección de los órganos’ según la noción de Kapp), ha ensanchado cada vez más la brecha entre el creador y sus creaciones, al punto de que estas últimas insertas en una dinámica que abarca otros ámbitos de la cultura (como la política y la economía) tiene todos los visos de autodeterminada. Con ello, pareciera que lo único que se ha logrado es haber conducido al ser humano en un rango de subordinación respecto de sus obras sin precedentes en la historia. ${ }^{43}$

\footnotetext{
${ }^{42}$ Es decir, disimulando su peligrosa tendencia, por ejemplo en lo que atañe a la ecología, al voluntarismo antropocentrista.

${ }^{43}$ El autor tuvo la oportunidad de profundizar en los temas de este trabajo, gracias a una generosa beca de investigación en la Universidad Heinrich Heine de Düsseldorf, concedida por el Servicio alemán de intercambio académico (DAAD) en 1999. Un agradecimiento especial por su hospitalidad y generoso apoyo a Alois Huning (Dusseldorf) y a Friedrich Rapp (Universidad de Dortmund).
} 
